\title{
OBSERVATIONS IN THE ASTEROID BELT WITH THE IMAGING PHOTOPOLARIMETER OF PIONEERS F AND G
}

\author{
C. E. KENKNIGHT \\ University of Arizona
}

\section{GENERAL DESCRIPTION}

Astronomical measurements of the brightness, color, and polarization of light from solar system bodies at phases not visible from Earth are planned for Pioneers $F$ and $G$. An imaging photopolarimeter (IPP) in the scientific payloads will make these measurements and produce line-scan color images of Jupiter. The IPP collects light with a $2.5 \mathrm{~cm}$ aperture telescope that is able to turn in the plane containing the spin axis. Data are taken in scans around the spin axis along a cone whose apex angle is periodically changed by stepping the telescope. The light is analyzed for polarization, divided into blue and red components, and detected by four photomultipliers having electron channel multipliers. Appendix A gives a summary of instrument characteristics.

\section{SCIENTIFIC EXPERIMENTS}

Modes of operation of the IPP include (1) standby, (2) zodiacal light, (3) polarimetry, and (4) imaging. Standby is not a data mode but power is applied to the instrument except for the detectors. Imaging is designed for the bright surface of Jupiter at encounter so that the instantaneous field of view $(0.0297)$ and dwell time per field of view $(1 \mathrm{~ms})$ are too small to be of interest in the asteroid belt. Both polarimetry and zodiacal light modes allow detection of objects brighter than $5 \mathrm{mag}$. (See app. B.) The polarimetry mode is more suited to stellar objects provided their sky position is known, whereas the zodiacal light mode is designed to measure the distribution of sky glow periodically between launch and Jupiter encounter. The sky accessible to the instrument is from $29^{\circ}$ from the earthward spin axis (limited by the communication antenna) to $170^{\circ}$ from that axis.

Observations in the zodiacal light mode will yield brightness and polarization maps of the sky. Subtraction of the maps made at different radial distances from the Sun will permit separation of the zodiacal light component from unresolved stars, galactic scattered light, etc. After separation, the radial distribution of zodiacal light scatterers can be inferred. Strips of the zodiacal 
light map are slightly overlapped because the field of view is $2^{\circ} .3$, the largest possible within the overall design, whereas the cone angle is stepped by 1.8 . Each scan contains 64 sectors of $5^{\circ} .6$ in the plane perpendicular to the spacecraft spin axis. The telemetry gives 1024 intensity levels for a measurement. The measurement cycle at each cone angle requires 20 rolls: 8 for data, 2 for calibration with the aperture closed or with a radioisotope-activated phosphor light source in view, and 10 for data readout. At a nominal $5 \mathrm{rpm}$ spin rate, an entire map requires about $5 \mathrm{hr}$ of observations. The operation is automatic after selection of the mode and of the detector sensitivity. Stepping can be inhibited or reversed by command to concentrate observations on a particular portion of the sky. Online data analysis will include displays of the data for a strip, which can be checked against prepared scans from star charts. Extensive observations to search for asteroidal object encounters will be avoided unless experience with other experiments suggests that our instrument is not apt to be damaged by small-particle impacts. In standby the IPP will point toward the antenna.

The field of view and the telescope step angle is 0.5 in the polarimetry mode. The detectors are sampled every 0.5 in this mode so the data fill the allowed memory in $70^{\circ}$ of roll angle. These data are read out in the remainder of a roll. Observations may begin from a roll angle at which a brightness threshold is exceeded or from commandable roll angles. The latter is more suited to the asteroid belt observations. The measurement cycle requires four rolls: (1) calibration lamp and dark current if sensitivity is low, or dark current if sensitivity is high; (2) clear aperture; (3) depolarizer; and (4) half-wave retarder plate to rotate linear polarization by $45^{\circ}$. Stepping proceeds automatically unless inhibited, and it may be reversed. A sector of roll angle $70^{\circ}$ long could be assembled into a map comparable to a zodiacal light map. The polarimetry map could be acquired at $4 / 3$ the zodiacal light mapping rate in cone angle but a search of the whole sky would be prohibitive in the polarimetry mode.

The probability of observing a numbered asteroid without highly accurate aiming is extremely small. Using the relation $B=B(1,0)+5 \log r d+F(\alpha)$ and the estimates $B(1,0)=10 \mathrm{mag}$ for the absolute magnitude of an asteroid with $20 \mathrm{~km}$ radius, $r=3 \mathrm{AU}$, and $F\left(90^{\circ}\right)=2.7 \mathrm{mag}$ as for the Moon (Harris, 1961), we may determine the miss distance $d \cong 1.0 \times 10^{-2} \mathrm{AU}$ giving an observed magnitude $B=5$. A computer search for numbered asteroids near the Pioneer $F$ trajectories was performed at NASA Ames Research Center (1970). The miss distances were $0.03 \mathrm{AU}$ or greater, but usually larger than $0.1 \mathrm{AU}$. The 20 asteroids reported usually have orbits that could not be reached without a change-of-plane maneuver by the spacecraft. One exception is 554 Peraga, whose inclination and node might permit a near passage. Its absolute magnitude $B(1,0)=9.4$ and it is the largest of the 20 asteroids. Aiming for this asteroid to within $0.01 \mathrm{AU}$ would make it detectable, but would add a significant constraint to the Jupiter transfer orbit. 


\section{APPENDIX A-SUMMARY OF PROPERTIES OF IPP}

\section{Modes of operation:}

1-Standby

2-Zodiacal light

3-Polarimetry

4-Imaging (for details see Gehrels, Suomi, and Krauss, 1972)

Telescope: Maksutov type with $2.5 \mathrm{~cm}$ aperture and $8.6 \mathrm{~cm}$ focal length Instantaneous field of view:

Mode 2: 2.3 square

Mode 3: $0^{\circ} .5$ square

Mode 4: 0.03 square

Spectral bandpass:

Blue: 390 to $500 \mathrm{~nm}$

Red: 595 to $720 \mathrm{~nm}$

\section{Polarization analyzer:}

Mode 2: symmetrical Wollaston prism

Mode 3: symmetrical Wollaston prism and achromatic half-wave retardaCalibration: tion plate

Mode 2: radioisotope-activated phosphor light source

Mode 3: solar diffuser, tungsten filament lamp and Lyot depolarizer

Detectors: Two dual-channel Bendix Channeltrons

Look angle step:

Mode 2: 1.8 every 20 rolls

Mode 3: 0.5 usually every four rolls

Mode 4: 0.03 every roll

(Step direction or "no step" commandable)

Look angle range: $10^{\circ}$ to $170^{\circ}$ from earthward spin axis (the antenna is seen from $10^{\circ}$ to $29^{\circ}$ )

Sampling angle:

Mode 2: complete roll at 512 bps or higher telemetry rate

Mode 3: $70^{\circ}$ of roll at $1024 \mathrm{bps}$ or higher telemetry rate

Mode 4: $14^{\circ}\left(29^{\circ}\right.$ at low sampling rate $)$ at 1024 bps or higher telemetry rate

(Start of sampling in modes 3 and 4: one of 64 commandable angles or automatic at an increase in light in excess of a threshold)

Analog/digital conversion:

Mode 2: four channels, 10 bits each, after each integration period of $1 / 64$ th of roll

Mode 3: four channels, 10 bits each, after each $16 \mathrm{~ms}$ integration

Mode 4: two channels, 6 bits each, alternate channels every $0.5 \mathrm{~ms}(1 \mathrm{~ms}$ at low sampling rate)

(Five temperatures, 10 bits each) 


\section{Telemetry:}

Mode 2: 50 bits status, 50 bits temperatures, 2560 (maximum) bits detector data every other roll

Mode 3: 50 bits status, 50 bits temperatures, 6044 (maximum) bits detector data every roll

Mode 4: 50 bits status, 6094 (maximum) bits detector data every roll

(Data buffered by spacecraft -data storage unit and sent in telemetry format D1 or D2)

(One analog word (standby status) in subcommutated science data)

Commands: 3, mode initiate; 4, telescope step control; 4, data sampling control; and 2, gain control

Size: 18 by 19 by $47 \mathrm{~cm}$

Mass: $4.2 \mathrm{~kg}$

Power: $3.5 \mathrm{~W}$

\section{APPENDIX B-CALCULATION OF THE SENSITIVITY OF THE IPP IN THE POLARIMETRY MODE}

The signal current at the cathode is $I=s S E h A$ where $s$ is the response at the effective wavelength relative to the peak response ( 0.88 in $B, 0.39$ in $R) ; S$ is the peak cathode response of the cathode (S-20 extended, about $45 \mathrm{~mA} / \mathrm{W}$ ); $E$ is the optical efficiency including reflectivities, obscuration, and a factor of 0.5 for polarization state $(0.18$ in $B, 0.23$ in $R) ; h$ is the object intensity in watts per square centimeter passed by the filter; and $A$ is the mirror area $\left(5.0 \mathrm{~cm}^{2}\right)$.

If $H$ is the solar flux at each filter (about $200 \mathrm{~W} / \mathrm{m}^{2}$ ), then

$$
\log h / H=0.4(M-m)
$$

where $M$ is the solar magnitude and $m$ is the object magnitude. Consider $m=5$ and $M=-26.9$. Then $h=3.4 \times 10^{-15} \mathrm{~W} / \mathrm{cm}^{2}$ and $I_{R} \cong I_{B}=1.2 \times 10^{-16} \mathrm{~A}$.

Shot noise is a lower estimate of the system noise

$$
I_{n}=\left(2 K q I \Delta f_{n}\right)^{1 / 2}
$$

where $K=1.4$ is a noise factor given by the supplier, $q$ is the-electron charge $1.6 \times 10^{-19} \mathrm{C}$, and $\Delta f_{n}$ is an effective noise bandwidth $\left(4 t_{s}\right)^{-1}$ for a system time constant $t_{s}$ and a single time constant noise filter. For the signal to rise to 0.999 of its steady-state value in this mode, $t_{s}=t_{i} / 6.9$, where $t_{i}$ is the time for the spacecraft to turn one field of view,

$$
t_{i}=\frac{0.46 \mathrm{deg}}{28 \mathrm{deg} / \mathrm{s}}=16 \mathrm{~ms}
$$

Therefore $\Delta f_{n}=108 \mathrm{~Hz}$ and $I_{n}=0.62 \times 10^{-16} \mathrm{~A}$. By comparison the dark current is about $0.05 \times 10^{-16} \mathrm{~A}$ in the blue tubes, and somewhat larger in the red tubes. 
Evidently the signal to noise ratio for a 5 mag object and one detector is $I / I_{n}=2$. Because the signal is divided between two pixels on the average, the recognition of a signal is somewhat poorer than this signal to noise ratio would indicate. But the use of four detectors during four rolls at a particular cone angle in this mode brings the signal to noise ratio to 8 . Thus a 5 mag object is barely recognizable.

\section{ACKNOWLEDGMENTS}

The University of Arizona (T. Gehrels) has overall responsibility for the program, Dudley Observatory (J. L. Weinberg) has responsibility for observations and analysis of the zodiacal light, and Santa Barbara Research Center is responsible for fabrication and testing of the instrumentation. We thank C. F. Hall and his staff at the Pioneer Project Office, NASA Ames Research Center, for their effective cooperation.

\section{REFERENCES}

Gehrels, T., Suomi, V. E., and Krauss, R. J. 1972, On the Capabilities of the Spin-Scan Imaging Technique. Space Research XII (ed., A. C. Strickland). Akad. Verlag. Berlin. Harris, D. 1961, Photometry and Colorimetry of Planets and Satellites. Planets and Satellites (eds., G. Kuiper and B. Middlehurst), pp. 272-342. Univ. of Chicago Press. Chicago.

NASA Ames Research Center. 1970, Pioneer F Asteroid Analysis. Pioneer Program Document PT-204.

[Editorial note: The Pioneer Mission to Jupiter is described in NASA SP-268.] 